sinusoidal external electric field. The combined effect of slip flow and electrokinetics was demonstrated on the velocity profile in confined geometries. The velocity profile was observed to be a function of both slip coefficient and external electric field. They observed that both these effects play an important role for flow inside the microchannel.

$\mathrm{Ou}$ and Rothstein [10] developed an ultra hydrophobic surface with micrometer-sized ridges (20-30 $\mu \mathrm{m}$ wide) placed 20-120 $\mu \mathrm{m}$ apart aligned in the flow direction. They demonstrated maximum $25 \%$ drag reduction for flow inside these channels. From $\mu$-PIV measurements, they showed the existence of slip flow in the air-water interface between the ridges, while the flow over the ridges obeys the no-slip condition. They attributed the existence of slip flow for drag reduction.

\section{Future Directions for Research}

The studies carried out so far have explained the slip flow phenomena to a great extent. However, the use of slip phenomena for the analysis of different fluid flow problems is limited. The effect of various flow parameters, i.e., roughness, wettabilty, polarity, and presence of nanobubbles or residual gases, on slip flow phenomena need to be conclusively and systematically studied. The effect of temperature and concentration gradient on slip flow parameters needs to be properly characterized based on both molecular dynamics simulation and experiments. The effect of slip flow on turbulence production mechanism needs to be established. The development of advanced micro-/nanomanufacturing and measurement technology is expected to facilitate the systematic study of these parameters.

Manipulation of conditions required for the generation of slip flow can have many practical applications. This will be beneficial in the development of drag reduction and mixing enhancement technologies. This is particularly important for micro- and nanotechnologies, where the pressure penalty is very high and mixing is difficult due to the difficulty in the generation of turbulent flow.

\section{Cross-References}

- Atomic Force Microscope (AFM)

- Molecular Dynamics Simulation Method

\section{References}

1. Choi C-H, Westin JA, Breuer KS (2003) Apparent slip flows in hydrophilic and hydrophobic micro channels. Phys Fluids 15:2897-2902

2. Cheng JT, Giordano N (2002) Fluid flow through nanometer-scale channels. Phys Rev E 65:1-5

3. Neto C, Craig VSJ, Williams DRM (2003) Evidence of shear-dependent boundary slip in Newtonian liquids. Eur Phys J E 12:S71-S74

4. Tretheway D, Meinhart C (2002) Apparent fluid slip at hydrophobic microchannel walls. Phys Fluids 14: L9-L12

5. Tsai P, Peters AM, Pirat C, Wessling M, Lammertink Rob GH, Lohse D (2009) Quantifying effective slip length over micropatterned hydrophobic surfaces. Phys Fluids 21:112002

6. Barrat J, Bocquet L (1999) Large slip effect at a nonwetting fluid-solid interface. Phys Rev Lett 82:4671-4674

7. Ullmanella U, Ho C (2008) Molecular effects on boundary condition in micro/nanoliquid flows. Phys Fluids 20:101512

8. Thompson PA, Troian SM (1997) A general boundary condition for liquid flow at solid surfaces. Nature 389:360-362

9. Yang J, Kwok DY (2003) Effect of liquid slip in electrokinetic parallel-plate microchannel flow. J Colloid Interface Sci 260:225-233

10. Ou J, Rothstein JP (2005) Direct velocity measurements of the flow past drag-reducing ultrahydrophobic surfaces. Phys Fluids 17:103606-103610

\section{Boundary-Element Method in Microfluidics}

Barbaros Cetin ${ }^{1}$ and Besim Baranoğlu ${ }^{2}$

${ }^{1}$ Mechanical Engineering Department, Bilkent

University, Ankara, Turkey

${ }^{2}$ Department of Manufacturing Engineering,

Atılım University, Ankara, Turkey

\section{Synonyms}

Boundary element method; Boundary integral approach 


\section{Definition}

The boundary element method (BEM) is a numerical method for solving partial differential equations which are encountered in many engineering disciplines such as solid and fluid mechanics, electromagnetics, and acoustics.

\section{Overview}

Boundary element method (or boundary integral method) is a numerical tool that is well applied to many linear problems in engineering. There are several advantages of the boundary element method (BEM) over other numerical methods (such as finite element method (FEM)) and some of which are (i) discretization and modeling of only the boundary of the solution domain, (ii) improved solutions in stress concentration problems, (iii) accuracy of the solution regarding that it is a semi-analytical method (the integral equation is obtained using exact solution of the corresponding linear problem; the numerical approach is necessary only for evaluating the resulting integrals), and (iv) the possibility of application to domains that extend to infinity in one or more dimensions. Especially, for problems that involve successive remeshing, the boundaryonly nature of the BEM presents a valuable property - since there is no domain discretization, remeshing is not an important issue. Also, the continuity and compatibility conditions within the solution domain are satisfied in an exact manner in the BEM (in the FEM, on the contrary, the continuity and compatibility conditions across the elements are satisfied in a numerical manner).

The major disadvantage of the BEM is that nonlinear or nonhomogeneous applications of the method are not common since the method requires predefined fundamental solutions, which are to be determined for the given specific problem, and for most nonlinear problems, there are no such fundamental solutions provided in literature. There are some techniques to overcome this disadvantage of the BEM, such as defining internal cells for volume integration (which destroys the boundary-only nature of the
BEM) or using dual reciprocity method (which requires a set of additional collocation points defined in the solution domain).

Considering fluid flow in microchannels, typical flow speed is low (resulting in very low Reynolds number) and the inertia forces are negligible (in magnitude) when compared with the pressure or the viscous forces; the flow can be considered as the so-called creeping flow [1]. The governing equation of the creeping flow is the Stokes flow, which is a linear partial differential equation suitable for solution with the BEM. The boundary element representation of such flow was first developed by Youngren and Acrivos [2] for the analysis of flow over 3D and axisymmetric rigid particles. Same authors then extended their work for similar flow over deformable bubbles [3], and later a similar study involving viscous drops was introduced by Rallison and Acrivos [4]. As a different approach, the motion of a rigid sphere moving normal to a deformable interface was investigated using boundary element method by Lee and Leal [5]. All boundary element formulations indicated above are direct, which means they are obtained using field variables which has physical meaning - like components of the velocity vector, traction on the surface, and pressure.

Manipulation of bioparticles within a microchannel is a key ingredient for many biomedical and chemical applications. A rigorous simulation of the particle motion including the effect on the particle presence on the field variables requires massive remeshing when finite difference, finite element, and finite volume-based numerical techniques are considered. Moreover, the determination of the forces induced on the particles requires the calculation of gradient of the field variables. Therefore, for an accurate calculation of gradient of field variables, fine mesh is required on the particle surface. This issue is problematic when a particle moves in the vicinity of the wall. The interaction of the particle with the wall when the particle is moving close to the wall is difficult to model. In many cases, it is compulsory to introduce a model to take into account the particle-wall interaction $[6,7]$. Since BEM does not require meshing 
within the flow field and the exact calculation of gradient of field variables, and considering the linearity of the governing equations, BEM is a preferable tool for the simulation of the bioparticles in a microchannel. In this entry, advantages and challenges of the BEM for the simulation of particle trajectory for a pressuredriven flow in a microchannel will be discussed, and some preliminary simulations results will be presented.

\section{Basic Methodology}

\section{Boundary Element Formulation of Flow Field}

The governing equations for the steady-state Stokes flow (assuming the inertial effects to be negligible) can be given as $-\nabla P+\mu \nabla^{2} \mathbf{u}+\rho \mathbf{g}$. $\mathbf{x}=0$

where $p$ is the pressure, $\mathbf{u}$ is the velocity vector, and $\rho \mathbf{g}$ is the body forces. The material properties are the viscosity, $\mu$, and the density, $\rho$, of the fluid. Defining a modified pressure as $P=p-$ $\rho \mathbf{g} \cdot \mathbf{x}$ where $\mathbf{x}$ represents the coordinates, the Stokes equation can be rewritten as $-\nabla P+$ $\mu \nabla^{2} \mathbf{u}=\mathbf{0}$. The hydrodynamic stress tensor can be written as $\sigma_{i j}=-\delta_{i j} p+\mu\left(\frac{\partial u_{i}}{\partial x_{j}}+\frac{\partial u_{j}}{\partial x_{i}}\right)$ from which the tractions on the boundary of the region can be obtained as $t_{i}=\sigma_{i j} n_{j}$ where $n_{j}$ are the components of the unit normal vector pointing to fluid side at the given point. The boundary element (BE) formulation in this case is straightforward [1], where for a fixed point $\mathbf{A}$ the integral equations in $2 \mathrm{D}$ can be given as $C_{i j}(\mathbf{A}) u_{j}(\mathbf{A})=\int_{C} G_{i j}(\mathbf{A}, \mathbf{P}) t_{j}(\mathbf{P}) \mathrm{d} S-$ $\int_{C} H_{i j}(\mathbf{A}, \mathbf{P}) u_{j}(\mathbf{P}) \mathrm{d} S$ which is referred to as the reduced boundary element equation (RBEE). It is necessary at this point to note that for the above equation and for all equations, Einstein's summation convention is in place which implies (if not explicitly defined otherwise) a summation over repeated index. In RBEE, $C_{i j}(\mathbf{A})=\delta_{i j}$ if $\mathbf{A} \in V, \delta_{i j} / 2$ if $\mathbf{A}$ is on a smooth boundary, and 0 if $\mathbf{A} \notin V$. The $2 \mathrm{D}$ first and second fundamental solutions of the Stokes flow, e.g., $G(\mathbf{A}, \mathbf{P})$ and $H(\mathbf{A}, \mathbf{P})$, that appear as kernels in the integrals in RBEE are given as $G_{i j}(\mathbf{A}, \mathbf{P})=\frac{1}{4 \pi \mu}\left[-\ln (r) \delta_{i j}+r_{i} r_{j}\right]$ and $H_{i j}(\mathbf{A}, \mathbf{P})=\frac{r_{i} r_{j} \partial r}{\pi r \partial n}$ where $r=r(\mathbf{A}, \mathbf{P})$ is the distance between the fixed point $\mathbf{A}$ and the varied point $\mathbf{P}$ and $r_{i}=r_{i}(\mathbf{A}, \mathbf{P})$ are the components of the unit vector directed from $\mathbf{A}$ to $\mathbf{P}$. The directional derivative $\frac{\partial r}{\partial n}$ can be obtained as $\frac{\partial r}{\partial n}=r_{i} n_{i}$.

The RBEE is exact in the sense that, if the integral equations can be solved, the solution will be the exact solution to the actual model. Yet, even with many assumptions and simplifications both on geometry of the problem and the boundary conditions, the solution to RBEE is, if not impossible, very hard to obtain. Therefore, a numerical solution is attempted. For this, the boundary of the solution region is divided into a predefined number of elements. For each element, there exist a number of computational nodes (will be called only "nodes"). For simplicity, without losing generality, in this discussion constant elements are employed. For constant elements, the computational node is at the midpoint of the element, and all field variables associated with the element are assumed to have a constant value that equals to the value at the node of the element. A simplified mesh with constant elements can be seen in Fig. 1 .

For the constant elements, since the field variables $u_{i}$ and $t_{i}$ are assumed to be constant

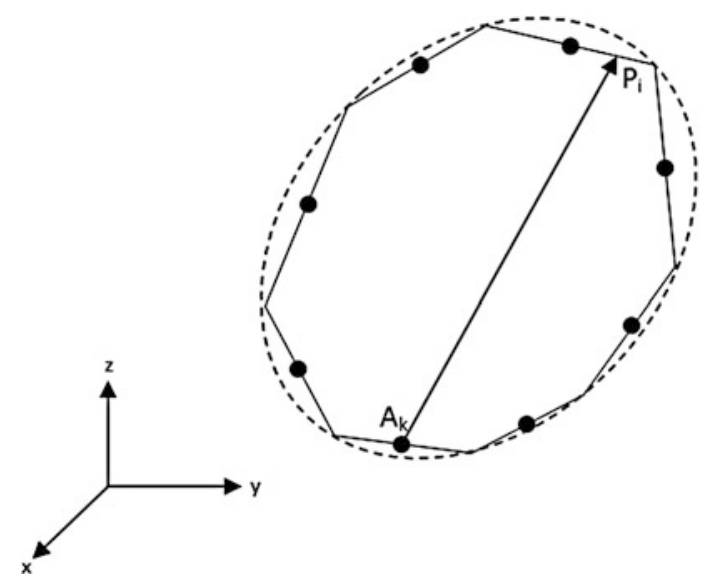

Boundary-Element Method in Microfluidics, Fig. 1 The constant element mesh for solution of the RBEE 
over the element, the integrals can be written as: $\int_{C_{k}} G_{i j}(\mathbf{A}, \mathbf{P}) t_{j}(\mathbf{P}) \mathrm{d} S=t_{j}\left(\mathbf{A}_{k}\right) \int_{C_{k}} G_{i j}\left(\mathbf{A}, \mathbf{P}_{k}\right) \mathrm{d} S$ and $\int_{C_{k}} H_{i j}(\mathbf{A}, \mathbf{P}) u_{j}(\mathbf{P}) \mathrm{d} S=u_{j}\left(\mathbf{A}_{k}\right) \int_{C_{k}} H_{i j}\left(\mathbf{A}, \mathbf{P}_{k}\right) \mathrm{d} S$ where $C_{k}$ is the portion of the boundary that corresponds to the $k$ th element with the node $\mathbf{A}_{k}$. When the RBEE is rewritten at all nodes $\mathbf{A}_{k} k=1 . . N$, with $G_{i j}^{l k}=\int_{C_{k}} G_{i j}\left(\mathbf{A}_{l}, \mathbf{P}_{k}\right) \mathrm{d} S$ and $H_{i j}^{l k}=\int_{C_{k}} H_{i j}\left(\mathbf{A}_{l}, \mathbf{P}_{k}\right) \mathrm{d} S$ and $u_{j}\left(\mathbf{A}_{l}\right)=u_{j}^{l}, t_{j}\left(\mathbf{A}_{l}\right)=t_{j}^{l}$ and considering the node on a constant element is on a smooth curve (line), one can obtain a linear set of equations which can be given as $\frac{1}{2} u_{i}^{l}+H_{i j}^{l k} u_{j}^{k}=G_{i j}^{l k} t_{j}^{k} \quad$ which can be rewritten (if diagonals $H_{i i}^{l l}$ are augmented with $\frac{1}{2}$ ) as $H_{i j}^{l k} u_{j}^{k}=G_{i j}^{l k} t_{j}^{k}$.

This gives a $2 N \times 2 N$ matrix equation which may be written as $\mathbf{H} \cdot \mathbf{u}=\mathbf{G} \cdot \mathbf{t}$ which then is solved in view of the boundary conditions. The imposition of boundary conditions in boundary element method is a simple task, which is achieved only by swapping the columns of the coefficient matrices. The aim is to collect all the known terms on the right-hand side (rhs) and all the unknowns to the left-hand side (lhs) of the equal sign. Therefore, for a selected couple $\left(u_{n}^{m}, t_{n}^{m}\right)$

which corresponds to the component in the $n$-direction of the $m^{\text {th }}$ element, if $t_{n}^{m}$ is known, no action is required since the known/unknown is in the right place; but if $u_{n}^{m}$ is known, then all terms containing this term should be swapped with the terms involving $t_{n}^{m}$, e.g., for each row, the corresponding equation is replaced with the equation below:

$$
-G_{i n}^{l m} t_{n}^{m}=-H_{i n}^{l m} u_{n}^{m}
$$

This corresponds to a column swap of the $(2 *(m-1)+n)$ th column of the coefficient matrices (by taking the negatives of the columns).

After the necessary column changes, the system of equations take the form

$\mathbf{K} \cdot \mathbf{x}=\mathbf{L} \cdot \mathbf{b}$ where now $\mathbf{x}$ represents the unknown vector and $\mathbf{b}$ represents the vector formed by the boundary conditions of the system. The solution can be obtained after evaluating the rhs multiplication, e.g., $\mathbf{K} \cdot \mathbf{x}=\mathbf{l}$.

\section{Simulation of Particle Trajectory}

Particle trajectory is the result of the interaction of the particle with the electric field and the flow field. To simulate the particle trajectories, there are two approaches. The first approach is the Lagrangian tracking method, which neglects the finite size of the particles and treats them as point particles and solves the field variables without the presence of the particles [8]. In this case, only the effect of the field variables on the particle is considered. The second approach is the stress tensor approach, which includes the size effect of the particle. In this approach, the field variables are solved with the presence of the finitesized particle, and the particle translates as a result of the interaction of the particle with the electric and flow field [8]. In each incremental movement of the particle, the field variables need to be resolved. The former approach is very simple and works good to some extent, and the latter approach is accurate yet computationally expensive.

\section{Lagrangian Tracking Method (LTM)}

In this approach, particles are assumed to be point particles, and the effect of the particle on the field variables is ignored; only the effect of the field variables on the particle is considered. The field variables are determined without the presence of the particles. The particle position $\boldsymbol{x}_{p}$ can be determined, by integrating the particle velocity together with the initial position:

$$
\mathbf{x}_{p}(t)=\mathbf{x}_{o}+\int_{0}^{t} \mathbf{u}_{p}(\tau) d \tau
$$

For a fixed frame of reference, the translational motion of a particle is governed by

$$
m_{p} \frac{d \mathbf{u}_{p}}{d t}=\mathbf{F}_{\mathrm{ext}},
$$

where $m_{p}$ is the particle mass and $F_{\text {ext }}$ is the net external force. In a microfluidic environment, one source of the external force is the drag force on the particle. Depending on the application, on top of the drag force, electrokinetic forces, magnetic forces, or acoustic forces may need to be 
included. Depending on the particle, Brownian motion may also be included. In LTM, the analytical expression for a spherical particle for a given force is used. The drag force on a spherical particle is given by

$$
\mathbf{F}_{\mathrm{drag}}=6 \pi \mu R\left(\mathbf{u}-\mathbf{u}_{p}\right),
$$

at the creeping flow limit, which is known as the Stokes law, where $\mathrm{R}$ is the particle radius, $\mathbf{u}$ is the fluid velocity, and $\mathbf{u}_{p}$ is the particle velocity (the analytical expressions for other forces can be found elsewhere [9]).

For the particle size considered in this study, the characteristic time scale of acceleration period of the motion is in the order of $10^{-4} \mathrm{~s}$ [8] which is much smaller than the time scale of the variation of the field variables. Therefore, the acceleration term can be safely neglected, and the velocity of the particle can be derived by equating the total external force to zero.

This approach is very simple and however has some limitations. LTM is valid if the particle size is small compared to the device dimensions, and in this version, it is valid for spherical particles. For the Stokes law to be valid, the particle needs to be several diameters away from the solid boundaries and the other particles (to model the hydrodynamic interaction of the particle with the wall, a correction factor needs to be introduced [6]) Moreover, if the disturbance of the flow field is significant due to the presence of the particle (e.g., the number of particles may be high within the domain or the size of the particle may be comparable with the microchannel size), the validity of the LTM is questionable. Since LTM does not include the presence of the particle, the simulation of the flow field can be performed with any standard software which handles the solution of PDEs, and the trajectory of the particles can be obtained at the postprocessing step.

\section{Stress Tensor Approach}

In this approach, the field variables are determined with the presence of the finite particle size. The resultant force on the particle can be obtained by integrating the appropriate stress tensor on the particle surface. In the case of a fluid flow, the resultant drag force on the particle can be determined by integrating the hydrodynamic stress tensor, which is given as

$$
\underline{\mathbf{T}_{h}}=-p \underline{\mathbf{I}}+\mu\left(\nabla \mathbf{u}+\nabla \mathbf{u}^{T}\right)
$$

over the particle surface. Once the resultant forces are given, Newton's second law of motion can be integrated to get the new position of the particle. This approach can be applied for any geometry, and particle interaction with the field and particle-particle interaction can be included. The drawback of this method is that it is computationally expensive. As the particle moves in the microchannel, the meshes need to be updated from time to time. Although BEM can be used for both approaches (LTM and STA), at this point BEM introduces a unique advantage for STA since in BEM only the boundaries of the domain are meshed. For an undeformable body, only the mesh on the particle surface needs to be moved in accordance with the appropriate forces. Utilizing this fact, Dustin and Luo $[10,11]$ implemented BEM to simulate the particle trajectory within a microchannel under the action of electrophoretic and electroosmotically driven flow field.

\section{Boundary Element Formulation of Particle Trajectory}

For this purpose, an incremental time stepping is suggested. At each time increment, the fluid flow will be assumed to be steady. This assumption holds if the particle dimensions are sufficiently small when compared with the characteristic dimensions of the problem (e.g., channel wall dimensions) and the change of location of the particle within one time increment is not significant. In this case, the inertial terms in the fluid flow equation can be neglected, and a steady-state assumption can be made.

In the analysis, further assumptions can be stated as the particle is bouyant, e.g., the net force acting on it (as it freely moves within the medium) is zero and the mass of the particle and the time rate of change of its velocity are 
sufficiently small that we may assume the inertial effects in the motion of the particle may be neglected.

Also, within the framework of this analysis, the particle is assumed to be rigid.

Recall the matrix equations of the BEE and how the boundary conditions are imposed on the system. The imposition of such boundary conditions requires, at all points of the defined boundary, the definition of one and only one of the couples $\left(u_{n}^{m}, t_{n}^{m}\right)$ or at least a combination of the two. When the motion of a particle in the fluid is considered neither the velocity components, $u_{i}$, nor the traction components, $t_{i}$, nor a combination of the two is known at a given element $m$. Rather, it is known that since the particle moves freely (e.g., free of forces), the net force over the surface will be zero. Also, the motion is a rigid body motion; therefore, all points on the particle surface will have a velocity that can be expressed as $u_{i}=u_{i}^{b}+\omega r \hat{t}_{i}$.

Here, $u_{i}$ represents the velocity at the particle boundary, $u_{i}^{b}$ are the velocity components of the particle center (translation), and $\omega$ is the angular velocity about the particle center. The distance between the particle center and the boundary point is given as $r$ and the components of the counterclockwise normal to the position vector form the center of the particle to the corresponding node is denoted by $\hat{t}_{i}$.

Considering the above discussion, three new unknowns can be introduced (in 2D): $u_{1}^{b}, u_{2}^{b}$, and $\omega$. All nodal velocities on the particle boundary will be a function of these three new unknowns. Assume now that the nodes $M$ to $N$ (which makes up $N-M$ nodes) belong to the particle. Then, for a node $m$ on the particle boundary, one can write the above rigid body motion equation with the emphasis that $r \rightarrow r^{(m)}$ gives the distance from the node to the particle center and $\hat{t}_{i} \rightarrow \hat{t}_{i}^{(m)}$ is the unit tangent at the given node. With this, the equations can be modified for a given row $(l, i)$ of the system of equations (which in fact belongs to the equation that is obtained by fixing the point $\mathbf{A}$ to $\mathbf{A}_{l}$ and in the $i$-direction) which in fact corresponds to the $(2 \times(l-1)+i)^{\text {th }}$ row. The modification would be
$\sum_{m=1}^{M-1} H_{i j}^{l m} u_{j}^{m}+\sum_{m=M}^{N} H_{i j}^{l m}\left(u_{i}^{b}+\omega r^{(m)} \hat{t}_{j}^{(m)}\right)=G_{i j}^{l m} t_{j}^{m}$

which implies the addition of three columns for the three unknowns in the $\mathbf{H}$ matrix. For the elements of the first two columns, we have $\sum_{m=M}^{N} H_{i j}^{l m}$ and for the elements of the third column, $\sum_{m=M}^{N} H_{i j}^{l k}\left(r^{(m)} \hat{t}_{j}^{(m)}\right)$

At this point, one can easily judge that all traction components for the nodes belonging to the particle are unknown. Therefore, a column swap of columns for the nodes $M$ to $N$ is required.

To make the system of equations solvable, three more equations should be stated. These three equations are obtained from the equilibrium conditions, e.g., the total force and moment on the particle boundary should be zero. Thus, the integrals relating the tractions to the total force and moment should add up to zero. This will bring in the required three more equations.

As the system of equations are solved, the rigid body motion parameters for the particle center will be obtained. With this velocity and the predefined time step, the particle may be moved to its new calculated location.

\section{Key Research Findings}

As a benchmark study, two-channel geometry and two-particle geometry are chosen as shown in Fig. 2. Fist geometry is a straight channel with a channel width, W, and a channel length, L. For the particle geometry, spherical (with a diameter d) and elliptical (with long-axis diameter, $\mathrm{d}_{\mathrm{l}}$, and short-axis diameter, $d_{s}$ ) particles are chosen, since many bioparticle-like bacteria and cells can be modeled with these two geometries. The particles are released initially from the location $\left(\mathrm{x}_{\mathrm{r}}, \mathrm{y}_{\mathrm{r}}\right)$ and the particle simulations are stopped when the particles reach $x=450 \mu \mathrm{m}$. The geometric parameters used in the simulations are given in Table 1. 
Boundary-Element Method in Microfluidics,

Fig. 2 Schematic drawing of the computational domain (a) straight microchannel (b) microchannel with hurdle
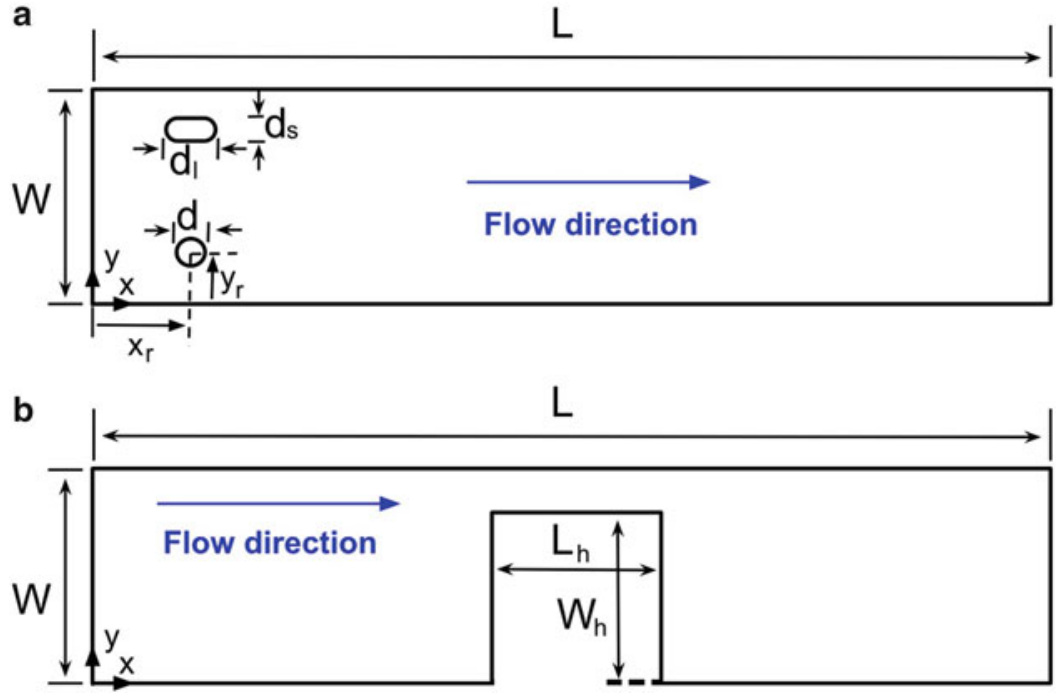

Boundary-Element Method in Microfluidics, Table 1 Geometric parameters used in the simulations

\begin{tabular}{llll}
\hline $\mathrm{L}$ & $500 \mu \mathrm{m}$ & $\mathrm{x}_{\mathrm{r}}$ & $50 \mu \mathrm{m}$ \\
\hline $\mathrm{W}$ & $100 \mu \mathrm{m}$ & $\mathrm{y}_{\mathrm{r}}$ & $10,20,30,50,70 \mu \mathrm{m}$ \\
\hline $\mathrm{L}_{\mathrm{h}}$ & $100 \mu \mathrm{m}$ & $\mathrm{d}$ & $10 \mu \mathrm{m}$ \\
\hline $\mathrm{W}_{\mathrm{h}}$ & $70 \mu \mathrm{m}$ & $\mathrm{d}_{\mathrm{l}}$ & $10 \mu \mathrm{m}$ \\
\hline & & $\mathrm{d}_{\mathrm{s}}$ & $6 \mu \mathrm{m}$ \\
\hline
\end{tabular}

In the simulations, the density of the fluid is taken as $1,000 \mathrm{~kg} / \mathrm{m}^{3}$, and the viscosity is taken as $0.001 \mathrm{~Pa} . \mathrm{s}$ which are the typical values for water (for bioparticle-based applications, the buffer solutions are water-based solutions). The particle is assumed to be buoyant which is the case for many bioparticles. At the inlet of the channel, uniform velocity of $300 \mu \mathrm{m} / \mathrm{s}$ is assigned. On the channel walls and the particle surface, no-slip boundary condition is applied, and zero pressure at the outlet of the channel is assigned. Typical mesh used in the simulations is shown in Fig. 3. 300 elements in the length direction, 50 elements in the width directions, and 32 elements on the particles are used for the straight channel. 250 elements on the top wall, 390 elements on the lower wall (finer mesh is implemented in the vicinity of the hurdle), and 50 elements on the inlet and the exit are used for the channel with the hurdle. The simulations were performed on an HP Z400 Workstation (Intel Xeon W3550, Quad core, $3.06 \mathrm{GHz}, 16 \mathrm{~GB}$ RAM). Although the total time step taken for each particle depends on the released point, it was observed that typical run-time for the simulation of a single particle varies between 1 and 2 min.

The particle trajectory of $10 \mu \mathrm{m}$, spherical particle is illustrated in Fig. 4. The particles are released from $\mathrm{y}_{\mathrm{r}}=10,30$, and $50 \mu \mathrm{m}$. For each time step of $0.25 \mathrm{~s}$, the orientation of the particle is shown in the figure. To demonstrate the rotation of the particle, one point on the particle is marked with red dot. The x-velocity and the angular speed of the particles are given in Fig. 4-b. Since it is a spherical particle in the Stokes flow, the x-velocity and the angular speed of the particles are steady. As the particle moves closer to the centerline, it travels with a higher velocity due to the parabolic nature of the velocity profile. Therefore, the x-velocity of the article increases as $\mathrm{y}_{\mathrm{r}}$ increases. As the particle moves closer to the channel wall, the symmetry of the flow at the upper and the lower surface of the channel distorts which results in a higher rotational speed. As a result, the angular speed of the particle increases as $y_{r}$ decreases. Since the velocity on the upper half of the particles is higher than the lower half, the rotation of the particles is in the clockwise direction. Due to the symmetry of the flow field, the particles released from $\mathrm{y}_{\mathrm{r}}=70$ and $90 \mu \mathrm{m}$ would have the same trajectory with a counterclockwise rotation. 
Boundary-Element Method in Microfluidics,

Fig. 3 Meshing of the computational domain
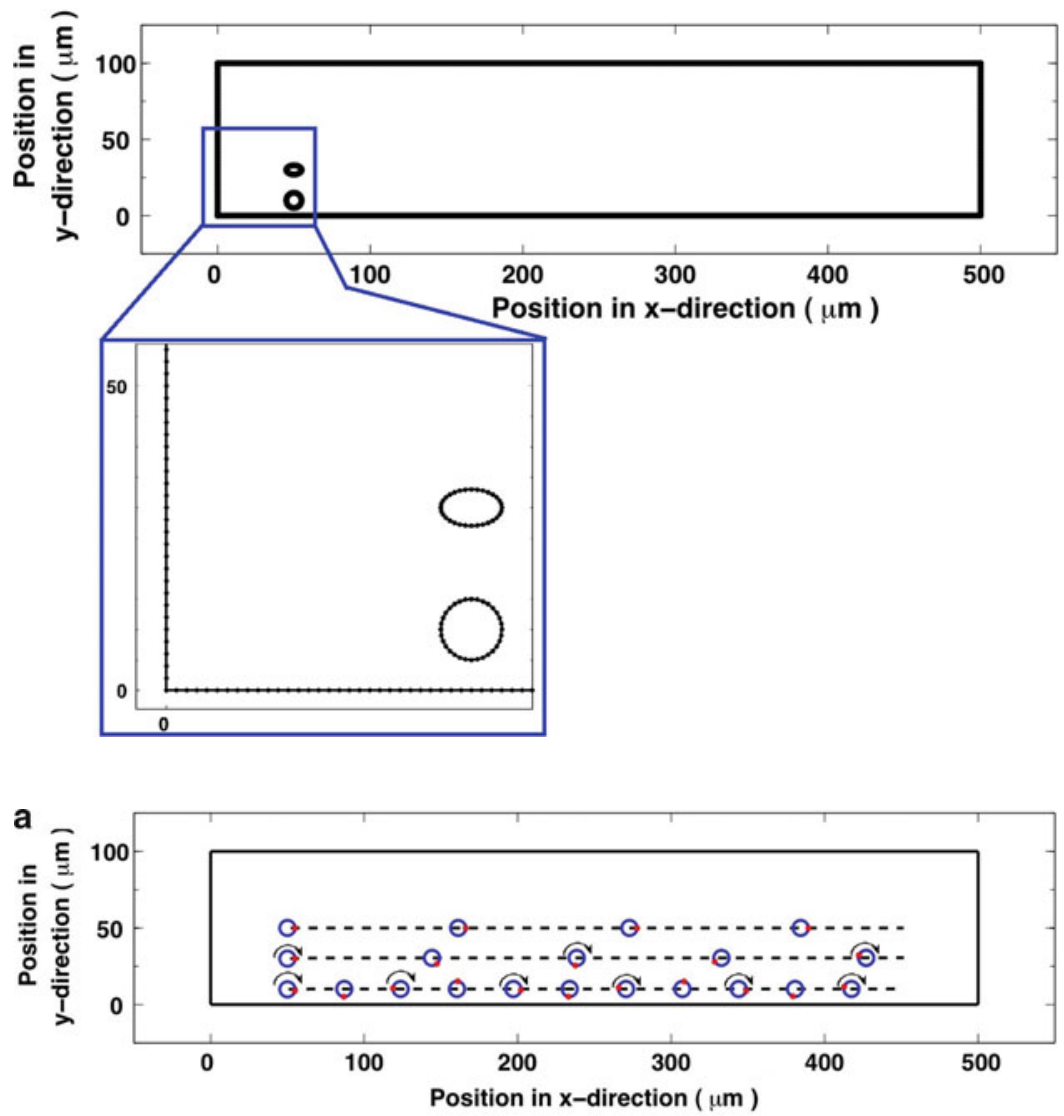

\section{Boundary-Element} Method in Microfluidics,

Fig. 4 (a) Motion of spherical particle in a microchannel (b) $\mathrm{x}$-velocity and angular speed of the spherical particle

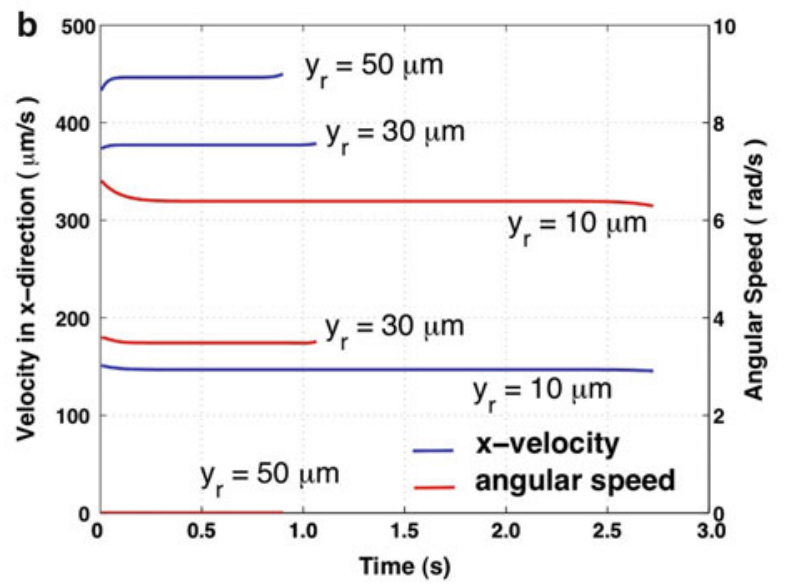

The motion of an elliptical particle in a straight channel is shown in Fig. 5. The rotation of the particle can also be seen, and again it is in the clockwise direction. The same conclusions for spherical particle are also valid for the elliptical particle. However, the major difference is the x-velocity and the angular speed of the particle. Especially for the angular speed, there exists a steady periodic behavior due to the geometry of the particle. The magnitude of the angular speed depends on the instant orientation of the particle. Moreover, even x-velocity has a steady periodic behavior for the particle moving near the wall. 
Boundary-Element Method in Microfluidics,

Fig. 5 (a) Motion of elliptical particle in a microchannel (b) $\mathrm{x}$-velocity and angular speed of the spherical particle
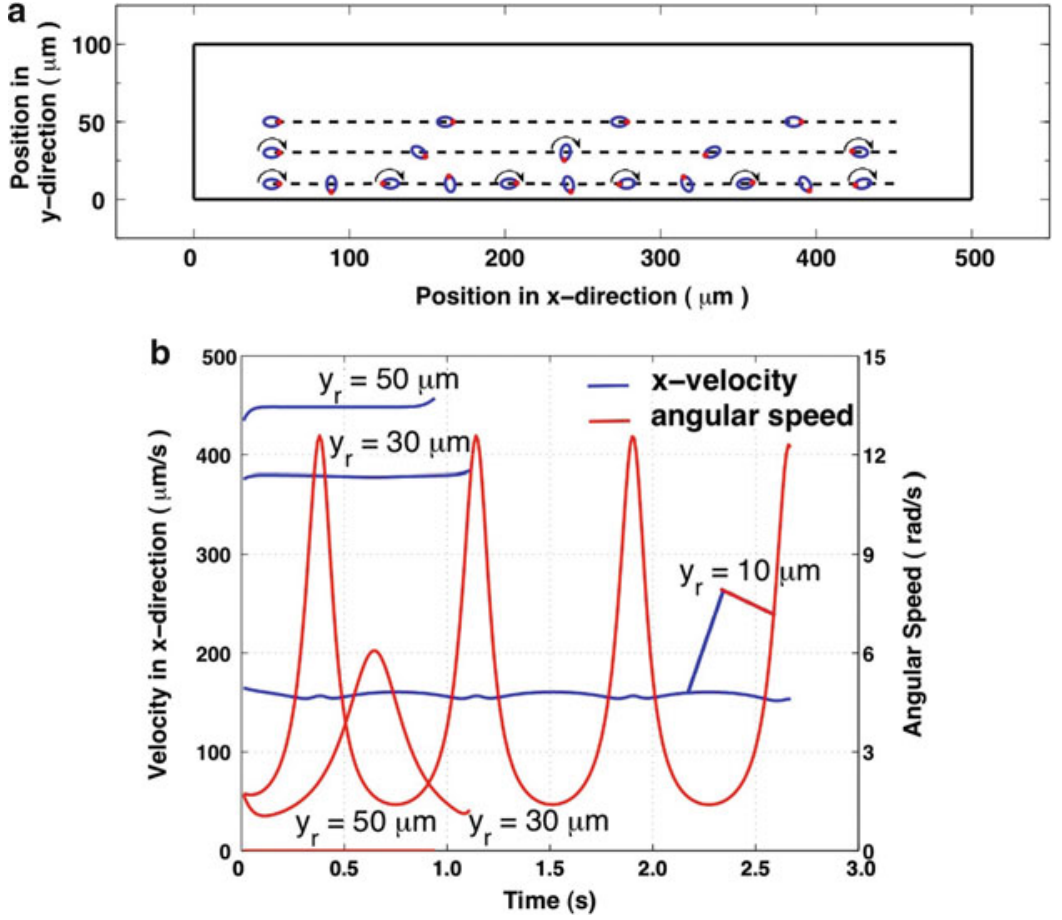

As a second benchmark problem, the motion of spherical and elliptical particles are analyzed in a channel with a hurdle at the middle. The results for spherical (Fig. 6) and elliptical particles (Fig. 7) are shown in the figures below. The rotation of the particles can also be realized. The particles realesed from $10 \mu \mathrm{m}$ follows a streamline which is different than that of $10 \mu \mathrm{m}$ after the hurdle due to hydrodynamic interaction of the particles at the corners and within the hurdle section. As the released location increases, this issue diminishes. Although, it is not simulated, the interaction of the particles with the corners has size dependence. So, the location of the particles after the hurdle depends on the size of the particle. BEM has clearly the ability to model this hydrodynamic interaction with the wall without any need for correction factor. The dependence of the equilibrium position after the hurdle on particle size is the key ingredient for the microfluidic devices for hydrodynamic separation of bioparticles. With the ability of BEM, this issue can be explored in details to come up with efficient microfluidics bioparticle separators.

\section{Future Research Directions}

BEM is an effective computational tool for microfluidic simulations. Especially, computational tools to simulate motion of particles in a microchannel are very crucial for the optimum design of the microfluidic devices. The implementation of BEM for this kind of problems is relatively new in this field. Therefore, there are many possible future research directions which can be summarized as follows:

- Considering the electrokinetic phenomena occurring in many microfluidic-based devices, such as electroosmotic pumping, electrophoresis, and dielectrophoresis, the governing equation is the Laplace equation which is in many cases also a linear partial differential equation. Electrokinetic forces may also be included in the BEM model together with the flow field. Similarly, inclusion of the acoustic and/or magnetic forces is also possible since the governing equations are in the linear form for many microfluidic applications. 
Boundary-Element Method in Microfluidics,

Fig. 6 Motion of spherical particle in a microchannel with hurdle (a) $\mathrm{y}_{\mathrm{r}}=10 \mu \mathrm{m}$, (b) $\mathrm{y}_{\mathrm{r}}=20 \mu \mathrm{m},(\mathbf{c})$ $\mathrm{y}_{\mathrm{r}}=50 \mu \mathrm{m},(\mathbf{d}) \mathrm{y}_{\mathrm{r}}=70 \mu \mathrm{m}$

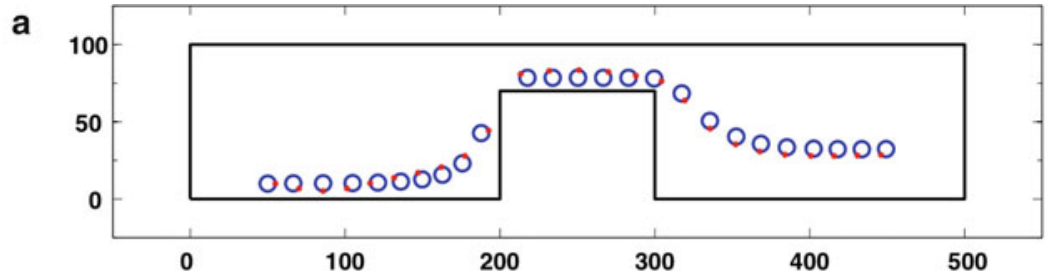

b

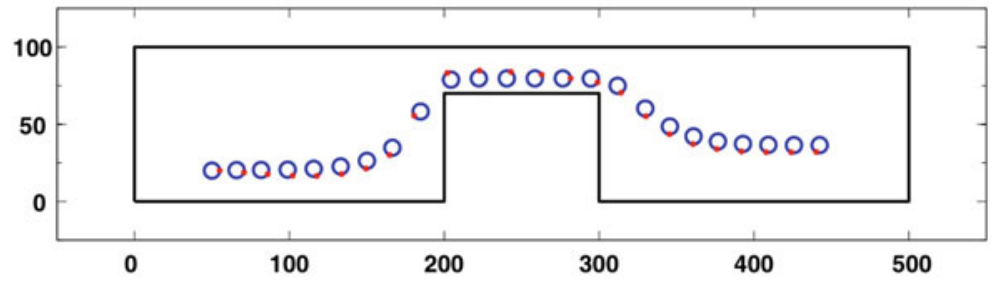

C
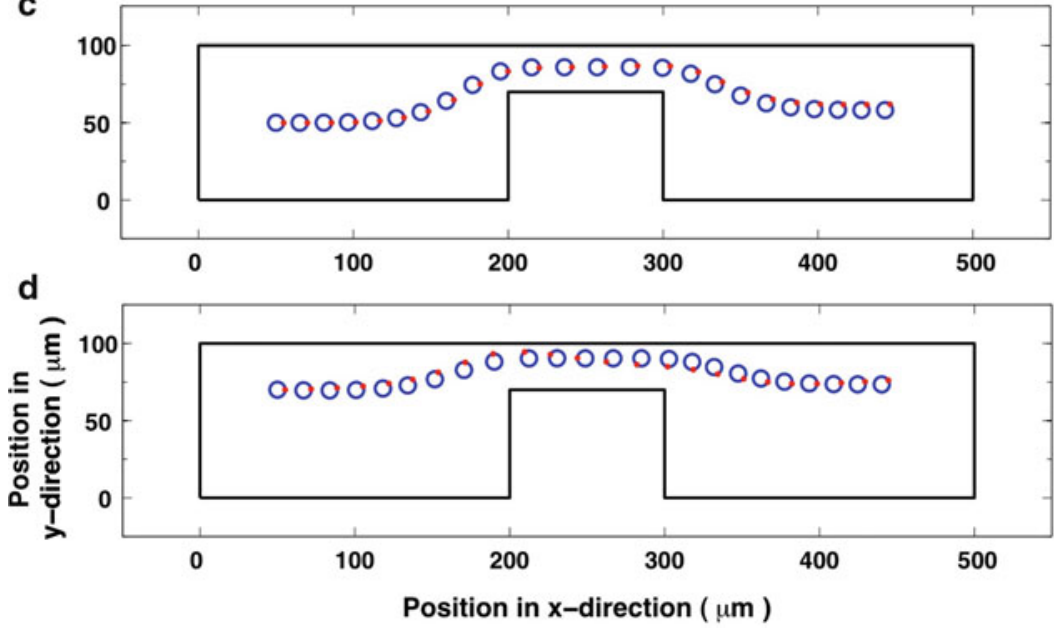

- Although neglection of the acceleration term for the particle is a reasonable approach, the neglection of the time-dependent term for the Stokes flow is problematic especially for nonspherical particles. Inclusion of the time derivative term for the flow field may result in more rigorous solution.

- $2 \mathrm{D}$ modeling is very common in microfluidic applications. Strictly speaking 2D modeling is appropriate for electroosmotic-based flow in which the flow profile is pluglike. For pressure-driven flows, a rigorous modeling requires 3D modeling. 3D modeling with BEM is a straightforward extension of the 2D model. Since the BEM discretizes only the boundary of the solution domain and no need for remeshing as the particle moves within the flow field, BEM can be implemented effectively even for 3D simulations.

- In BEM, all the elements of the system matrices can be determined independently, and the resulting linear system of equations has a fully populated coefficient matrix. Referring to these issues, the algorithm is highly parallelable. Moreover, by exploring the nature of the linear system resulting in microfluidic applications, effective iterative numerical algorithms can be developed.

- In many microfluidic applications, the number concentration of the bioparticles can be high, and the particle-particle interaction may be important when predicting the trajectory of particles. Introduction of many particles in a microchannel is a straightforward extension of the current model. Again with the 
Boundary-Element Method in Microfluidics,

Fig. 7 Motion of elliptic particle in a microchannel with hurdle (a) $\mathrm{y}_{\mathrm{r}}=10 \mu \mathrm{m}$, (b) $\mathrm{y}_{\mathrm{r}}=20 \mu \mathrm{m},(\mathbf{c})$ $\mathrm{y}_{\mathrm{r}}=50 \mu \mathrm{m}$, (d) $\mathrm{y}_{\mathrm{r}}=70 \mu \mathrm{m}$ a

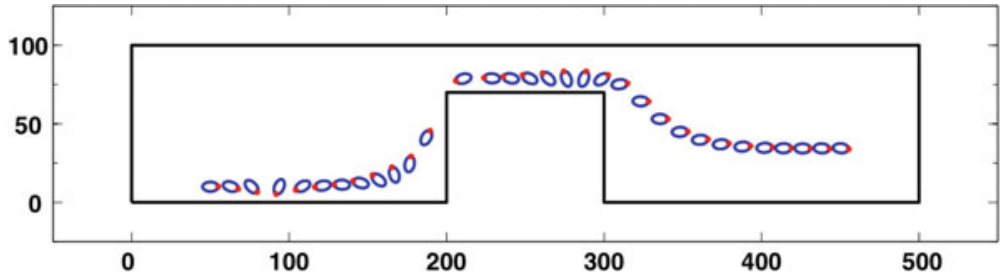

b

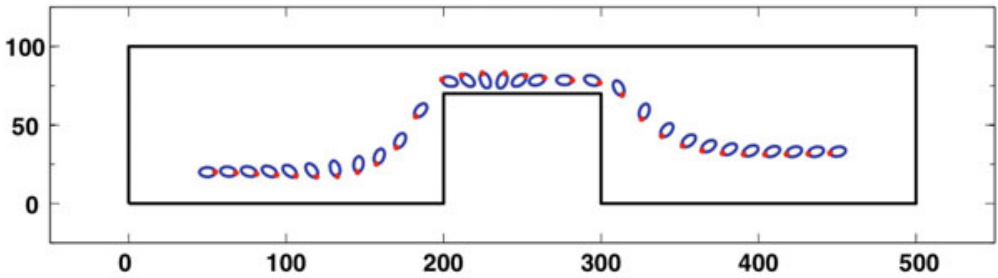

C
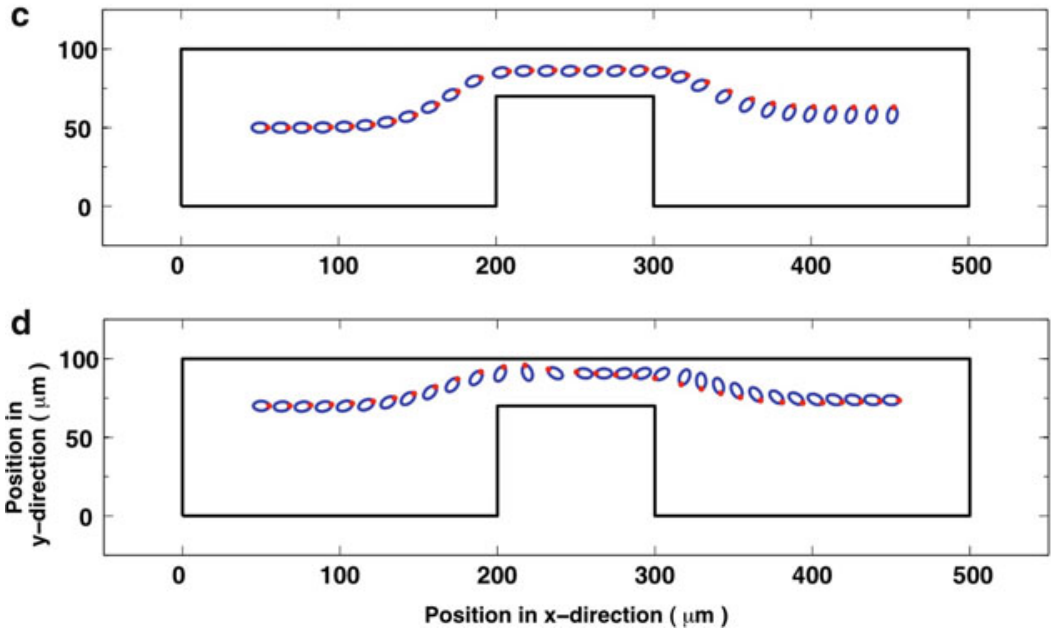

advantages associated with BEM, simulation of many particles can be computed in an effective manner.

- In the case of biomedical applications, bioparticles are highly deformable bodies. Although BEM is suitable for linear systems, nonlinear modeling has also been implemented for plasticity problems. Therefore, the inclusion of the deformation of the bioparticles is possible with BEM formulation which is very valuable for microfluidic applications.

\section{Cross-References}

- Boundary Element Method and Its Applications to the Modeling of MEMS Devices

\section{References}

1. Wrobel LC (2002) Boundary element method. Applications in thermo-fluids and acoustics, vol 1. Wiley, Chichester

2. Youngreen GK, Acrivos A (1975) Stokes flow past a particle of arbitrary shape: a numerical method of solution. J Fluid Mech 69:377-403

3. Youngreen GK, Acrivos A (1976) On the shape of a gas bubble in a viscous extensional flow. J Fluid Mech 76:433-442

4. Rallison JM, Acrivos A (1978) A numerical study of the deformation and burst of a viscous drop in an extensional flow. J Fluid Mech 89:191-200

5. Lee SH, Leal LG (1982) The motion of a sphere in the presence of a deformable interface. Part 2: numerical study of the translation of a sphere normal to an interface. J Colloid Interface Sci 87:81-106

6. Al Quddus N, Moussa WA, Bhattacharjee S (2008) Motion of a spherical particle in a cylindrical channel using arbitrary Lagrangian-Eulerian method. J Colloid Interface Sci 17:20-630 
7. Ai Y, Joo SW, Jiang Y, Xuan X, Qian S (2009) Pressure-driven transport of particles through a converging-diverging microchannel. Biomicrofluidics 3:022404

8. Çetin B, Li D (2011) Dielectrophoresis in microfluidics technology. Electrophoresis 32:2410-2427, Special issue on dielectrophoresis

9. Bruus H (2008) Theoretical microfluidics. Oxford University Press, New York

10. House DL, Luo H (2010) Electrophoretic mobility of a colloidal cylinder between two parallel walls. Eng Anal Bound Elem 34(5):471-476

11. House DL, Luo H (2011) Effect of direct current dielectrophoresis on the trajectory of a non-conducting colloidal sphere in a bent pore. Electrophoresis 32:3277-3285

\section{Bragg Diffraction Equation or Bragg's Law}

\section{Definition}

If a crystal is irradiated with $\mathrm{x}$-ray radiation most of the radiation passes through unhindered. But some part of the radiation will be deflected by the crystal, which is called Raylight scattering and can be visualized on photographic plates. But the equation can only be achieved (and thereby the reflection be observed), if the pitch length (which is although the phase shift of the radiation before and after the deflection) is an integer multiple of the wavelength. The Bragg diffraction equation was developed by William Henry Bragg and his son William Lawrence Bragg in 1912.

\section{Brownian Motion in Microfluidics and Nanofluidics}

Pramod Mathai

Department of Bioengineering, University of Maryland, College Park, MD, USA

\section{Synonyms}

Brownian diffusion

\section{Definition}

The temperature of a fluid is a measure of the average kinetic energy of the fluid molecules. Thus, all fluids above absolute zero consist of molecules that constantly collide with each other and with any other object suspended in the fluid. Even at thermodynamic equilibrium in the fluid (zero average heat flow), these collisions impart a random motion to every particle in the fluid that is termed as Brownian motion - named after Robert Brown who first observed such motion in pollen grains suspended in a fluid.

\section{Overview}

Albert Einstein was the first [1] to describe the cause of this motion in a comprehensive way that related the prevalent kinetic theory viewpoint with the random-walk theory-based description that was coming into vogue at the time. Quantifying the extent of Brownian motion provided an early proof of the existence of molecules and was also recognized as a way to model a variety of applications - for example, in stock markets and astrophysics - wherein an entity is subject to random interactions with its environment. In this entry, the theory, measurement, and applications of Brownian motion in a fluidic setting are described, and the current avenues of research - which probe beyond the canonical view - are discussed.

\section{Key Research Findings: Theory, Measurement, and Applications of Canonical Brownian Motion}

A defining characteristic of canonical Brownian motion is the linear dependence of a particle's mean squared displacement with respect to time. This dependence can be understood with a simple model: the random motion of a particle that is constrained to move either left or right on a line with equal probability by a distance $l$ in every time interval $\delta t$. The particle's displacement $r_{N}$ at the end of $N$ time intervals is given by $r_{N-1} \pm l$ 
with equal probability. If the experiment of taking the $N$ th step was repeated over a large number of runs, the mean displacement $\left\langle r_{N}\right\rangle$ over the runs satisfies $\left\langle r_{N}\right\rangle=\left\langle r_{N-1}\right\rangle=0$ for all $N$ by mathematical induction (since both left and right displacements are equally probable). However, the mean squared displacement $\left\langle r_{N}^{2}\right\rangle$ satisfies $\left\langle r_{N}^{2}\right\rangle=\left\langle r_{N-1}^{2}\right\rangle+l^{2}$ (since the cross term $\left\langle \pm 2 r_{N-1} l\right\rangle$ equals zero). By induction, $r_{N}^{2}=N l^{2}$, i.e., the mean squared displacement is proportional to the total number of time steps $N$. For the more general case of a $k$ - dimensional Brownian motion of a freely diffusing sphere in a fluid, one can derive a similar linear dependence of the mean squared deviation with respect to time [2]: $\left\langle r^{2}(t)\right\rangle=2 k D t$, where $k$ is 1,2 , or $3,\left\langle r^{2}(t)\right\rangle$ is the mean squared displacement in time $t$, and $D$ is the diffusion coefficient that depends on the size of the sphere and the fluid's temperature and viscosity. Figure 1 shows a simulation of Brownian motion in two dimensions.

The diffusion coefficient can be derived for a body of any shape or size by first computing its hydrodynamic drag and then using the fluctuation-dissipation (F-D) theorem to infer the diffusion coefficient. The central idea here is that both the Brownian motion (the fluctuation) of the body and the hydrodynamic drag (the dissipation) experienced by it in a fluid have their origin in the motion of fluid particles around the body and thus both quantities should be related. A clear introduction to the implications of the F-D theorem (a broad result that sheds light on varied phenomena like vacuum fluctuations and Johnson noise) for Brownian motion is available in Gillespie [2] and is critical to a deeper understanding of Brownian motion. The central argument is as follows. The forces on a particle of mass $M$ moving in one dimension with instantaneous velocity $V(t)$ at time $t$ in a fluid can be resolved into a dissipative component and a zero-mean fluctuating component yielding the following equation by using Newton's law:

$$
M \frac{d V(t)}{d t}=-\gamma V(t)+f \Gamma(t)
$$

where $\gamma$ (the dissipative component) is the drag coefficient of the particle and $\Gamma(t)$ (the fluctuation component) is a zero-mean Gaussian white noise force whose magnitude is set by the constant $f$. By using the principle of equipartition of energy (that relates the average kinetic energy of the particle to the surrounding temperature) along with Eq. 1, one can deduce that $f=\left(\gamma k_{\mathrm{B}} T\right)^{1 / 2}$, where $k_{B}$ and $T$ are the Boltzmann constant and

\section{Brownian Motion in Microfluidics and Nanofluidics,}

Fig. 1 Simulation of two-dimensional Brownian motion of a sphere: The initial and final position of the sphere is shown by the green and red circle, respectively. Although the mean deviation is zero (since every direction is equally probable), the mean squared deviation is nonzero (4Dt, in two dimensions)
Two dimensional Brownian motion of a sphere

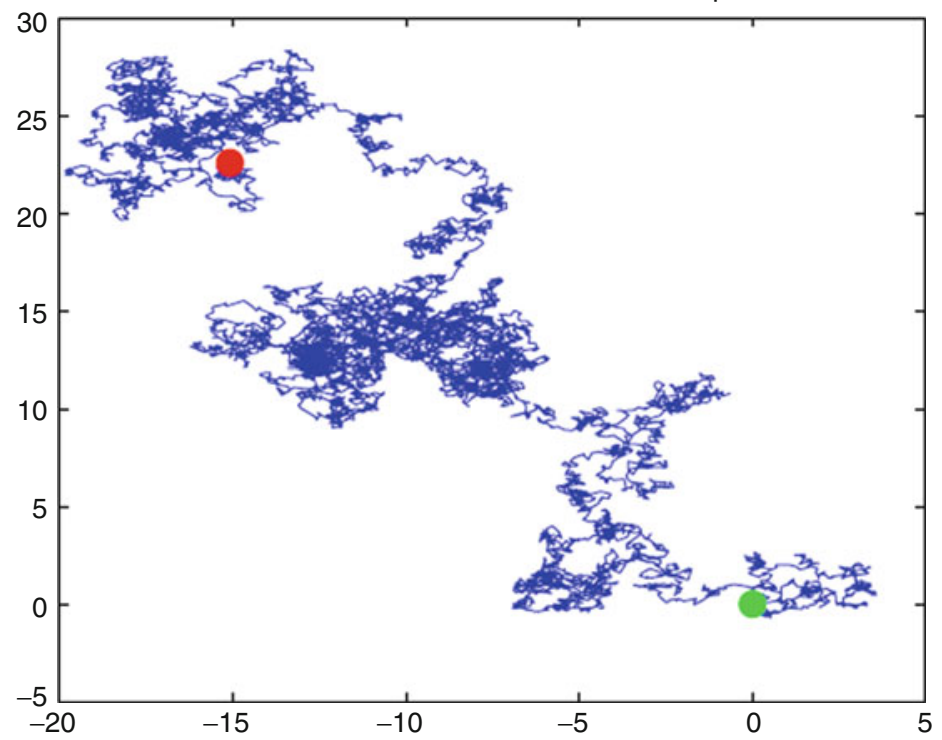


temperature of the fluid, respectively. This is an expression of the F-D theorem as applied to Brownian motion since it relates the strength of the fluctuation $f$ with that of the dissipation $\gamma$. A further analysis that assumes that consecutive kicks imparted to the body by the surrounding fluid are uncorrelated in magnitude or direction can be used to derive that $\left\langle X^{2}(t)\right\rangle=2 D t$, where $X(t)$ is the displacement of the body for large enough times $t$ (here $X(t+d t)=X(t)+V(t) d t$ for an infinitesimal time $d t$ ) and the diffusion coefficient $D$ equals $\frac{k_{\mathrm{B}} T}{\gamma}$. Thus, a knowledge of the drag coefficient $\gamma$ of the particle allows one to estimate the diffusion coefficient. Such a relation holds for each degree of freedom of the particle's motion. In particular, the rotational diffusion coefficient of the particle can be related to its rotational drag coefficient by working out the consequences of the fluctuation-dissipation theorem for the mean squared angular displacements of the particle.

Thus, the task of calculating the diffusion coefficient of a particle is reduced to the task of computing its drag coefficient $\gamma$. For complicated particle shapes, one can simulate Stokes flow around the particle, using a finite element simulation, to calculate $\gamma$. The simplest diffusion translational and rotational diffusion coefficients are those of a spherical particle; its one-dimensional translational diffusion coefficient is $\frac{k_{\mathrm{B}} T}{6 \pi \mu r}$ and its one-dimensional rotational diffusion coefficient is $\frac{k_{\mathrm{B}} T}{8 \pi \mu r^{3}}$, where $\mu$ is the dynamical viscosity of the fluid and $r$ is the radius of the sphere.

The established method for experimentally estimating the diffusion coefficient of a particle makes use of the linear dependence of its mean squared displacement (MSD) with respect to time (another method that involves a maximum likelihood estimate (MLE) that is optimal with respect to an information-theoretic limit has been proposed recently [3]). However, noise in the measurement of the displacements, due to optical and instrument constraints, complicates this estimation. Frequently though, the mean squared measurement noise $\left\langle X_{\text {meas }}^{2}(t)\right\rangle$ is well approximated to be additive [4] and satisfies $\left\langle X^{2}(t)\right\rangle=2 D t+$ $\left\langle X_{\text {meas }}^{2}(t)\right\rangle$. Thus, a linear regression model is still suitable and is used to estimate the diffusion coefficient of a colloidal particle experimentally in the following way:

1. A sample particle is immobilized on a slide and the measurement noise $\left\langle X_{\text {meas }}^{2}(t)\right\rangle$ is calculated with the help of a particle-tracking algorithm.

2. In a separate experiment, a sample particle is allowed to freely diffuse in the fluid, while its center of mass is estimated at regular time intervals (30 ms suffices for a micron-sized particle in water at room temperature) using the particle-tracking algorithm.

3. Using the time series data of the particle positions, the MSD for a given length of time (averaged over several time intervals of the same length) is estimated.

4. A linear regression model of the MSD versus time is fit that against the model $\left\langle X^{2}(t)\right\rangle=2 D t$ $+\left\langle X_{\text {meas }}^{2}(t)\right\rangle$, with the slope yielding the diffusion coefficient and the intercept yielding the measurement noise.

The rotational diffusion coefficient can be analogously estimated by tracking the angular mean squared displacements. Several issues - for example, drift (in addition to diffusion) due to any unresolved forces on the particle, blurring of a fast-diffusing particle's image in the camera frame - can complicate the estimation of the diffusion coefficient, requiring a different approach for an accurate measurement (like the MLE algorithm mentioned above).

Due to the ubiquitous nature of Brownian motion in microfluidics, the ability to constrain it has wide-ranging applications in sensing and directed assembly. The constraint is provided by imposing an appropriately shaped external potential on the particle's motion. For example, if the purpose is to trap a colloidal particle positionally, then the potential well should be deep enough so that the particle cannot escape the well due to the random Brownian perturbations experienced by it. Such potentials typically employ forces that depend on the particle's material properties, for example, optical and electrical. An alternate approach that does not rely on the particle's material properties but instead involves shaping the potential well by altering the flow surrounding 
the particle was recently demonstrated to both translate and rotate particles that are subject to Brownian perturbations [5]. Directed motion of an asymmetrically shaped "ratchet" particle can also be achieved by ensuring that the particle is placed in a thermal gradient so that the net effect of the surrounding fluid molecules randomly impinging on the particle is to push it preferentially in a desired direction [6].

\section{Future Directions: Beyond the Canonical View}

The theory and measurement techniques pertaining to the canonical Brownian motion of a particle in a fluid have been outlined above. We now describe two interesting areas of current research that probes deeper - motion at short length and timescales and motion when the step lengths and waiting times between steps are part of a broad-tail distribution.

\section{Ballistic to Brownian Transition: What Happens at Confined Length and Timescales}

The linear dependence of the mean squared deviation with respect to time is a defining hallmark of Brownian motion. However, this dependence is a result of the averaging over a large number of "kicks" received by the particle from the surrounding fluid molecules. Since such kicks impart a ballistic motion to the particle, a natural question arises: What does the motion of the particle look like at short times and distances after each kick, i.e., during the transition from ballistic to Brownian motion?

The mean squared formula for diffusion tells us that a micron-sized particle will diffuse a distance of around $1 \mathrm{~nm}$ in $1 \mu$ in water at room temperature. But this formula ignores the inertia of the particle and of the fluid displaced by it. It has been shown both theoretically and experimentally (see, e.g., [7] and references therein) that at such short timescales, both the inertia of the particle and the fluid displaced by it matter and that the central assumption in the mean squared formula - that of uncorrelated "kicks" - does not hold. In a short enough time, when a particle moves a short distance along a linear path due to its inertia, the flow around it is altered in such a way that subsequent kicks from the surrounding fluid molecules are more likely to come in the direction opposing its immediate ballistic motion, making subsequent kicks correlated to the immediately preceding ones. It takes a nonzero amount of time - around $10 \mu \mathrm{S}$ for a microparticle in water - to observe this transition from ballistic to Brownian motion, i.e., for kicks to be truly uncorrelated. Within this time interval, the mean squared displacements do not follow the Stokes-Einstein estimate. In Lukic et al. [7], the motion of a single microparticle that was constrained to move within an optical trap in water clearly shows this transition from ballistic to Brownian motion. Such singleparticle experiments have been repeated in other environments (including air) and confirm theoretical predictions.

The short-timescale theory and experiments mentioned above assume that a particle is surrounded by molecules from a single fluid. Efforts to understand the short-timescale motion in a heterogeneous environment - different fluids or a fluid and solid - are also important. Nanoscale Brownian motion in heterogeneous environments is important, for example, when studying the motion of DNA molecules while they are being sequenced as they get pulled through nanoscale pores in a solid (see, e.g., the work by Cees Dekker's lab in the Netherlands) - a sequencing method that is rapidly gaining viability on a commercial scale. A recent study of confined diffusion of fluid molecules within few nanometers of a solid [8] showed using molecular simulations that diffusion in confined spaces slows down due to two effects. First, steric hindrance of the fluid molecules due to their geometric confinement near a solid boundary inhibits their motion. Second, the potential energy wells created by the electrical forces exerted by the solid molecules on the liquid molecules can be deep enough that the latter's diffusional motion is inhibited.

Future research will include extension of these results to important technological applications. For example, in the case of the DNA-nanopore 
sequencing technique, one can envision that research in confined diffusion can aid in choosing an appropriate set of molecules in the vicinity of the pore and in designing an appropriate pore geometry so that Brownian motion of the DNA, as it is pulled through the pore, is suppressed. This will result in more accurate sequencing due to an increase in the signal-to-noise ratio of the electrical readout signal during the sequencing step.

\section{Anomalous Brownian Motion}

The relation $\left\langle X^{2}(t)\right\rangle=K t^{\alpha}$ between mean squared displacement and time presents a range of possibilities for varying $\alpha$, in which canonical Brownian motion $(\alpha=1)$ and ballistic motion $(\alpha=2)$ represent just two possibilities. All cases apart from $\alpha=1$ are clubbed under the label "anomalous Brownian motion" and encompass a range of interesting phenomena from biology to electronics [9]. The value of $\alpha$ is determined by two quantities - the variance in the step size $\sigma_{l}$ and the mean waiting time $\mu_{t}$ between consecutive steps - as discussed below.

In the random-walk description of Brownian motion, each step is considered to be uncorrelated with the last. However, their variance $\sigma_{l}$ is finite and so is $\mu_{t}$, the mean waiting time between steps. With these restrictions, it can be shown that regardless of the specific probability distributions from which the step size and time between steps are drawn, the motion is canonical, i.e., $\alpha=1$. However, if one relaxes the restriction on waiting times, i.e., allow the waiting times to be arbitrarily large, then one can see that the resulting motion must be slower than Brownian. Indeed, it can be shown [10] that the resulting motion, termed "sub-diffusive," follows the law $\left\langle X^{2}(t)\right\rangle=K t^{\alpha}$ with $\alpha<1$. Such motion is common in "sticky diffusion" of protein molecules on a cell surface where the molecule can chemically bind to the surface and can only move again after relatively long waiting time when it receives a perturbation of sufficient strength [9].

The opposite phenomenon, termed "superdiffusion," can also occur. For example, in the case when the mean waiting time is finite and the variance in the step length is arbitrarily large, the motion $\left\langle X^{2}(t)\right\rangle=K t^{\alpha}$ with $\alpha>1$ will result. Although the mathematical notion of an arbitrarily large step size is physically unrealistic, it has been shown that with appropriate restrictions on the maximum attainable velocity [10], one can indeed describe super-diffusive phenomena that have been observed in nature [9].

Future work, especially in cellular processes where molecules are always chemically interacting with their environment and hardly ever diffusing freely, will need to go beyond identifying that a process is anomalous - the root cause of the anomaly needs to be understood. The underlying mathematics, relating the mean step lengths and waiting times to the kind of anomaly, are clues leading to the root cause.

\section{Cross-References}

Biomolecular Adsorption in Microfluidics

- Boltzmann Equation

- Micro- and Nanoscale Gas Dynamics

- Micro- and Nanoscale Gas Fluidics

- Particle Manipulation Using Ultrasonic Fields

\section{References}

1. Einstein A (1905) On the movement of small particles suspended in stationary Liquids required by the molecular-kinetic theory of heat. Ann D Phys $17: 549$

2. Gillespie DT (1993) Fluctuation and dissipation in brownian motion. Am J Phys 61(12):1077

3. Michalet X, Berglund A (2012) Optimal diffusion coefficient estimation in single-particle tracking. Phys Rev E 85:061916

4. Crocker JC, Grier DG (1996) Methods of digital video microscopy for colloidal studies. J Coll Interf Sci 179:298

5. Mathai $P$ et al (2013) Simultaneous positioning and orientation of single nano-wires using flow control. RSC Adv 3:2677

6. Astumian RD (1997) Thermodynamics and kinetics of a brownian motor. Science 276:917

7. Lukic B et al (2005) Direct observation of nondiffusive motion of a brownian particle. Phys Rev Lett 95:160601

8. Matsubara H, Pichierri F (2012) Mechanism of diffusion slowdown in confined liquids. Phys Rev Lett 109:197801 
9. Klafter J, Sokolov IM (2005) Anomalous diffusion spreads its wings. Phys World 18, 29-32

10. Metzler R, Klafter J (2000) The random walk:s guide to anomalous diffusion: a fractional dynamics approach. Phys Report 339(77):1

\section{Bubble Dynamics in Microchannels}

Satish G. Kandlikar

Rochester Institute of Technology, Rochester, NY, USA

\section{Synonyms}

Bubble growth; Confined bubble growth; Explosive boiling in microchannels

\section{Definition}

Bubble dynamics in a microchannel refers to the nucleation of a bubble on heated flow channel walls and its subsequent interaction with the single-phase or two-phase flow field. Subcooled flow boiling refers to the boiling process with the mean flow enthalpy below the saturated liquid enthalpy at the local pressure, while saturated flow boiling refers to the boiling process with the mean flow enthalpy at or above the saturated liquid enthalpy but below saturated vapor enthalpy at the local pressure.

\section{Overview}

The formation and subsequent growth of a vapor bubble on a heated wall covered with a liquid is controlled by the forces arising from the excess pressure inside the bubble, the surface tension forces at the liquid-vapor interface and at the contact line formed by the interface at the heater surface, and the inertia forces resulting from the motion of the flow as well as the interface. The resistance to the phase change process at the liquid-vapor interface is quite small in comparison to the conduction resistance in the liquid surrounding a bubble and is generally neglected in analyzing the bubble dynamics.

\section{Basic Methodology}

The bubble dynamics during flow boiling in microchannels has been studied using highspeed video imaging and numerical techniques. The nucleation of a bubble and its growth leading to a confined bubble, vapor plug, and annular flow have been analyzed under flow boiling studies. The research has primarily been conducted with water and several refrigerants such as R-123 and FC72 as the working fluids.

\section{Key Research Findings}

Nucleation and Bubble Growth. Liquid enters a microchannel in a subcooled state and experiences a rise in temperature, while the local pressure falls due to frictional pressure drop along the channel in the flow direction. At some point downstream, the local saturation condition is reached. However, bubble nucleation will not occur immediately at this location as a certain degree of wall superheat is needed to activate a cavity according to the conditions originally suggested by Hsu [1]. The effect of the receding contact angle and the local flow field in front of a bubble was incorporated, and the following equation is recommended [2] for nucleation of minimum and maximum cavity radii, $r_{\mathrm{c}, \min }$ and $r_{\mathrm{c}, \max }$ :

$$
\begin{aligned}
& \left\{r_{\mathrm{c}, \min }, r_{\mathrm{c}, \max }\right\}=\frac{\delta_{t} \sin \theta_{\mathrm{r}}}{2.2}\left(\frac{\Delta T_{\mathrm{Sat}}}{\Delta T_{\mathrm{Sat}}+\Delta T_{\mathrm{Sub}}}\right) \\
& {\left[1 \mp \sqrt{\left.1-\frac{8.8 \sigma T_{\mathrm{Sat}}\left(\Delta T_{\mathrm{Sat}}+\Delta T_{\mathrm{Sub}}\right)}{\rho_{\mathrm{V}} h_{\mathrm{LV}} \delta_{t} \Delta T_{\mathrm{Sat}}^{2}}\right]}\right.}
\end{aligned}
$$

where $\delta_{t}=k / h ; k$ - thermal conductivity of the fluid, $\mathrm{W} /(\mathrm{mK}) ; h$ - single-phase heat transfer coefficient prior to nucleation; $\theta_{r}-$ receding contact angle of the bubble interface on the 